\title{
Investigation on Microstructure and Optical Property of Nanocrystalline Silicon Thin Film
}

\author{
Li-Qiang Guo*, Jian-Ning Ding, Guang-Gui Cheng, Zhi-Yong Ling and Zhong-Qiang Zhang
}

Micro/Nano Science \& Technology Center, Jiangsu University, 212013, Zhenjiang, China

\begin{abstract}
Nanocrystalline silicon (nc-Si:H) thin films were deposited by capacitive coupled radio-frequency plasma enhanced chemical vapor deposition (RF-PECVD) system with direct current (DC) bias applied. Raman, XRD and ultraviolet-visible transmission spectra were employed to investigate their microstructure and optical properties, respectively. Both the crystalline volume fraction and the average crystalline size increase with the substrate temperature. With the increase of silane concentration, the crystalline volume fraction increases, while the average crystalline size decreases. With the increase of the radio frequency (RF) power or the DC negative bias voltage, the crystalline volume fraction and the average crystalline size increase firstly, then decreases. Finally, the optical band gaps were discussed in detail.
\end{abstract}

Received on 03-09-2014 Accepted on 22-09-2014

Published on 30-10-2014

Keywords: PECVD, process conditions, optical property, microstructure.

\section{INTRODUCTION}

Great attentions have been paid to hydrogenated nanocrystalline silicon thin films (nc-Si:H) for promising applications in thin film photovoltaic cells due to its superior low energy photon absorption and optical stability against light soaking [1]. So far, great progress has been taken on the preparation, microstructure and properties of nc-Si:H thin film [2]. Generally, this material could be described as a twophase-mixed material [3], that is, silicon nanocrystallites are embedded with a-Si:H network. In addition, microstructure and physical property can be modified by changing process parameters [4]. Several deposition techniques have been established to prepare nc-Si:H films [5], including ion beam mixing, HW-CVD and PECVD deposition [6]. PECVD appears to be a promising deposition method because of the higher deposition rates and suitability for scaling up in large area industrial applications. PECVD process can be controlled by changing the substrate temperature, the RF power, the silane concentration and the DC negative bias voltage. The impacts of these parameters on nc-Si:H film's properties are mutual. Therefore, by keeping other factors constant, it is necessary to investigate the effect of one factor on its properties systematically. In this paper, the substrate temperature, the RF power, the silane concentration and the DC negative bias voltage in turn and solely were changed mainly, and effects of process

"Micro/Nano Science \& Technology Center, Jiangsu University, 212013, Zhenjiang, China; Tel: +086 13775533727; Fax: +086 0511-88791548;

E-mail: guoliqiang@uje.edu.cn, liqiangguo1984@163.com conditions on their microstructure and optical properties of nc-Si:H are investigated, systematacially.

\section{EXPERIMENTAL DETAILS}

Nc-Si:H thin films for solar cells were prepared using silane $\left(\mathrm{SiH}_{4}, 5 \%\right.$, dilution with $\left.\mathrm{H}_{2}\right)$ and hydrogen $\left(\mathrm{H}_{2}, 99.9999 \%\right)$ by changing the substrate temperature, RF power, the silane concentration and the DC negative bias voltage in turn and solely. The samples were deposited on glass substrates (Corning 7059) for subsequent measurement. To reduce pollution source, the glass substrates were cleaned by boiling with hydrogen peroxide and ammonia water for $10 \mathrm{~min}$. Prior to each deposition, the substrates and the deposition chamber were baked for one hour at $250{ }^{\circ} \mathrm{C}$ to remove any water vapor adsorbed on the substrates surface and the based pressure was taken out to $1.6 \times 10^{-4} \mathrm{~Pa}$. The nanostructure characteristics of samples were investigated with Raman spectroscopy and XRD. The Raman spectra were fitted by Gauss function into TO mode vibration peak of amorphous silicon at $480 \mathrm{~cm}^{-1}$ and TO mode vibration peak of crystalline silicon at $520 \mathrm{~cm}^{-1}$. The optical band gap was obtained from ultravioletevisible (UV-vis) transmission spectra.

\section{RESULTS AND DISCUSSION}

The composition of nc-Si:H thin film is considered to silicon particles embedded in amorphous intergranular boundary, as shown in the inset of Figure 1a. For nc-Si:H thin film, Raman scattering is inadequate to provide unambiguous information 

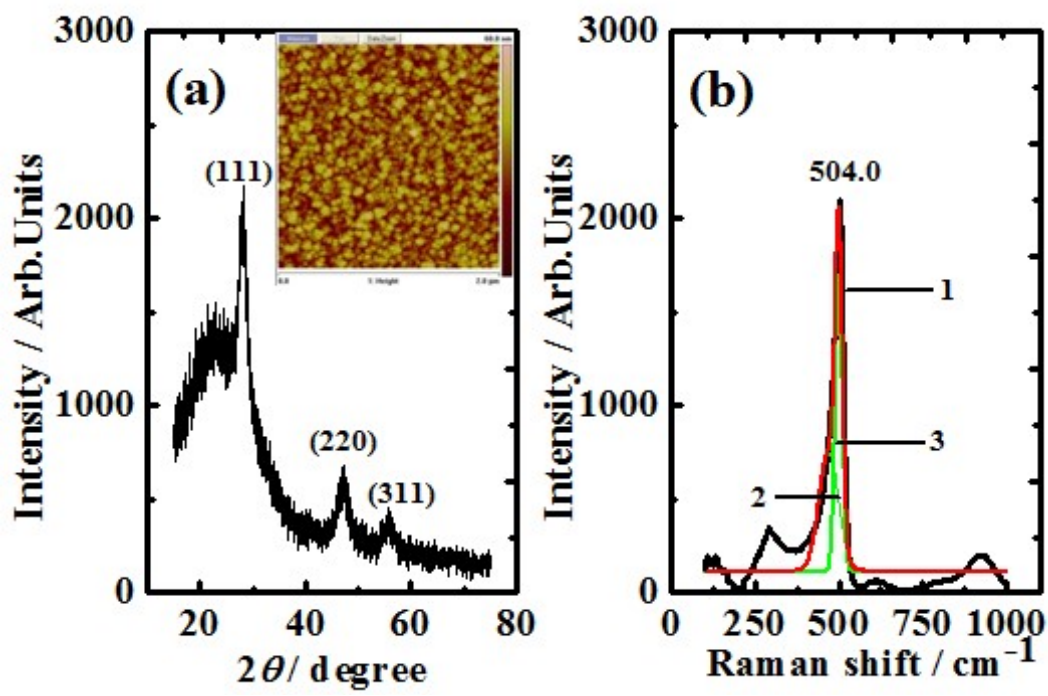

Figure 1: (a) The XRD spectra of a typical nc-Si:H thin film, inset in Fig is surface topography, (b) The Raman spectra of a typical nc-Si:H thin film.

about the crystallite size, the distribution and crystalline fraction in nc-Si:H thin films, additional data regarding to the structure of the films should be obtained by other techniques [7], such as XRD or SEM. The XRD spectrum of a typical ncSi: $\mathrm{H}$ film deposited at the temperature of $280^{\circ} \mathrm{C}$, RF power of $200 \mathrm{~W}$, the silane concentration of $2.5 \%$ and the DC negative bias voltage of $150 \mathrm{~V}$ is shown in Figure 1a. It is found that most of the diffraction peaks can be assigned to the (111), (220), (311) [8]. Before the (111), there is a big shoulder peak in XRD spectrum. This indicates that the growth of some crystalline grains is still far from completeness. The average crystallite size $d$ is calculated from FWHM of peak (111) using the Debye Scherer's formula [9]:

$$
d=\frac{k \lambda}{\beta \cos \theta}
$$

Where $k$ is a constant $(=0.94)$ [9], $\beta$ is the FWHM and $\lambda$ is the wavelength of the X-ray $\left(\mathrm{Cu}_{\mathrm{ka}}\right)(\lambda=0.154 \mathrm{~nm}$ in the present study); $2 \theta$ is the location of the diffraction peak.

The crystalline volume fraction $X_{c}$ is defined as the intensity ratio between the $520 \mathrm{~cm}^{-1}$ component and the $480 \mathrm{~cm}^{-1}$ component, as shown in Figure $\mathbf{1 b}$, and can be caculated by :

$$
X c=\frac{I_{c}}{I_{c}+m I_{\alpha}}
$$

Where $I_{c}$ and $I_{a}$ are integrated intensities of crystalline silicon and amorphous phase respectively, and $m$ is the fitting coefficient which can be expressed as [11]:

$$
m=0.1+\exp \left(-\frac{d}{25}\right)
$$

The crystalline volume fraction and the average crystalline size as a function of the substrate temperature are shown in
Figure 2a. RF power of $200 \mathrm{~W}$, the silane concentration of $0.1 \%$ and the DC negative bias voltage of $150 \mathrm{~V}$ are kept constants, respectively. When increasing the substrate temperature from $200{ }^{\circ} \mathrm{C}$ to $260{ }^{\circ} \mathrm{C}$, the crystalline volume fraction and the average crystalline size increase from $48.6 \%$ to $52.0 \%$ and $2.05 \mathrm{~nm}$ to $2.79 \mathrm{~nm}$, respectively. Due to the driving effect of the concentration gradient, the reactive groups would appear with a directional flow during the nc$\mathrm{Si}: \mathrm{H}$ thin film's growth. And this directional flow can be described by diffusion theory. As to the one-dimensional case, the diffusion flux $J$ is proportional to the concentration gradient of the reactive groups, that is:

$$
J=-D \frac{d n}{d x}
$$

Where, the negative sign "-" indicates that the diffusion flow directs from the large concentration space to the small concentration space. Scale coefficient $D$ is called as diffusion coefficient, and in equilibrium conditions it can be expressed as:

$$
D=D_{0} \exp \left(-\frac{E}{k T}\right)
$$

Where, $E$ is the self-diffusion activation energy. $D_{0}$ is the preexponential coefficient. $k$ is the Boltzmann constants. $T$ is the substrate temperature. Based on the formula (5), the diffusion coefficient of the reactive groups is closely related with the substrate temperature. Under the low substrate temperature, the density of hydrogen atoms and the diffusion coefficient of the reactive groups are relatively low. The reactive groups get not enough activation energy to find a stable nucleation sites, thereby, the order growth of the microstructure of nc-Si:H thin film is inhibited [12]. When increasing the substrate temperature, the environment of hydrogen released would be improved and the activity ability of the reactive group 
adsorbed is enhanced, and the number of hydrogen atoms which cover on the film's surface would be reduced [13]. The reactive silicon groups $\left(\mathrm{SiH}, \mathrm{SiH}_{2}\right.$, and $\left.\mathrm{SiH}_{3} \ldots \mathrm{SiH}_{n}\right)$ can get enough activation energy to improve their mobility on the film surface, and they are likely to spread to a stable nucleation position for their growth. Therefore, increasing the substrate temperature is helpful to film's crystallization. In addition, the reactive groups will get enough energy to adjust the bond angles during the combination on the cluster's surface, which results in the formation of the strong compound bond that promoting the grains to grow. In particular, when two or more adjacent clusters meet, the smaller one can adjust within a certain range to enhance the mergeing of clusters.

The crystalline volume fraction and the average crystalline size as a function of RF power are shown in Figure $\mathbf{2 b}$. For all of these samples, the temperature, the silane concentration and the DC negative bias voltage are kept constant, and are $280{ }^{\circ} \mathrm{C}, 0.1 \%, 150 \mathrm{~V}$, respectively. When increasing $\mathrm{RF}$ power from $100 \mathrm{~W}$ to $225 \mathrm{~W}$, the crystalline volume fraction increases from $47.4 \%$ to $52.1 \%$, then decrease to $48.9 \%$. The average crystalline size achieves to the maximum value of $2.48 \mathrm{~nm}$ at RF power of $150 \mathrm{~W}$, and then it decline. As we all know, RF source is one of the energies for glow discharge to decompose the reactive gas. It directly dominates the kinetic energy of the reactive radical decomposed from the reactive gas. The appropriate RF power provide kinetic energy for the radicals in the chamber, this would contribute to its growth on the substrate surface. But $[\mathrm{H}]$ etch must be also controlled accurately; otherwise it would affect the deposition rate and quality of nanocrystalline. When increasing RF power, the number and the energy of $[\mathrm{H}]$ would increase greatly, so that the etch rate of $[\mathrm{H}]$ is almost close to the deposition rate of the silicon film's deposition, making the film deposition rates continually decrease. When RF power is too large, due to the heavy collision between the plasma and the growth surface, the film will be damaged, therefore the number of the defects increase. And the crystalline volume fraction and the average crystalline size decrease.

The crystalline volume fraction and the average crystalline size as a function of the silane concentration are shown in Figure 2c. The RF power, the temperature and the DC negative bias voltage are kept at $200 \mathrm{~W}, 280{ }^{\circ} \mathrm{C}, 150 \mathrm{~V}$, respectively. With the increase of the silane concentration, the average crystalline size in the nc-Si:H thin film turns out an increasing trend, while the crystalline volume fraction decreases. Generally, it is believed that if both the average crystalline size and the crystalline volume fraction of the film are too large or too small, the film would be transformed into polycrystalline silicon thin film or amorphous/microcrystalline silicon thin film. And then it will lose the specificity properties of nano material. Therefore, either too large silane concentration or too small silane concentration is not suitable for the formation of nc-Si:H microstructure. The silane concentration is corresponding to the size of the hydrogen dilution ratio. And based on the ion bombardment model, during the growth of nc-Si:H thin film, $\mathrm{SiH}_{\mathrm{n}}$ transporte onto the film's surface and move to the right energy position, then deposite to form crystal growing point. Finally, it becomes a strong Si-Si bond. During the nucleation, if weak Si-Si bond appears in some direction, the crystalline growth will stop in
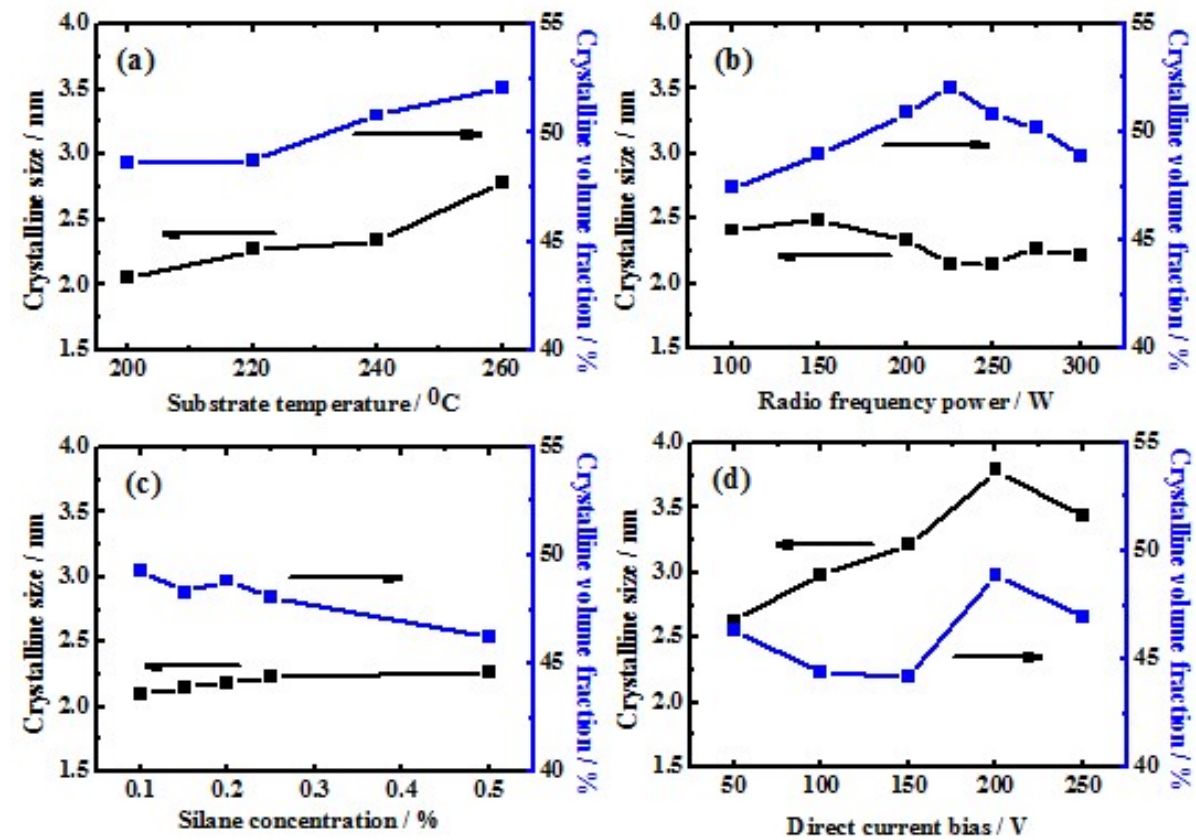

Figure 2: (a) The crystalline volume fraction and the average crystalline size as functions of substrate temperature for depositing nc-Si:H thin films, (b) The crystalline volume fraction and the average crystalline size as functions of RF power for depositing nc-Si:H thin films, (c) The crystalline volume fraction and the average crystalline size as functions of silane concentration for depositing nc-Si:H thin films, (d) The crystalline volume fraction and the average crystalline size as functions of DC bias for depositing nc-Si:H thin films. 
this direction, and the weak Si-Si bond will be etched by hydrogen particles gradually. If the silane concentration is relatively large, the number of the reactive group which reach the surface of film's growth in per unit is also quite large, so that the distance between the adjacent crystal nucleus is much smaller than the critical nucleation distance, therefore they cannot form the desired silicon nuclei, or the number of the desired silicon nuclei is small. At the same time, a large number of the reactive groups $\mathrm{SiH}_{\mathrm{n}}$ promotes few nucleus growth, and result in the increasing of average crystalline size. But many reactive groups have no chance to react, they will be mutually merged, and eventually attached to the surface of the films. Therefore, the silane concentration is one of the most critical parameters to achieve film crystallization [14].

The crystalline volume fraction and the average crystalline size as a function of the DC negative bias voltage are shown in Figure $\mathbf{2 d}$. The RF power, the temperature and the silane concentration are kept at $200 \mathrm{~W}, 280^{\circ} \mathrm{C}, 0.1 \%$, respectively. With the increase_of the DC negative bias voltage from $50 \mathrm{~V}$ to $200 \mathrm{~V}$, the crystalline volume fraction decreases from $46.3 \%$ to $44.2 \%$, and then increases to $48.9 \%$. While the average crystalline size increases from $2.63 \mathrm{~nm}$ to $3.79 \mathrm{~nm}$, and then decreases to $3.43 \mathrm{~nm}$. The DC negative bias voltage effectively controls the energy and the number of the reactive group $\mathrm{SiH}_{\mathrm{n}}$ on the substrate surface. Thereby, applying an appropriate DC negative bias voltage can make the reactive groups which have the appropriate energy on the gas-solid interface to nucleate and grow. However, when the DC negative bias voltage is small, the reactive energy is insufficient, and many reactive groups deposited with accumulation states and they composed amorphous components. When increasing the DC negative bias voltage, the activity of the reactive group increases. And when bonding between the groups, they can adjust the bond angle to form a stable and order microstructure, and the crystalline composition of the film will be increased significantly. However, as the ion bombardment energy increaseing, the internal stress of nc-Si:H thin film also increases, resulting in a lot of defects between the lattice and transfer crystalline state to amorphous state. In addition, the DC negative bias voltage can directly control the energy and momentum of $\left[\mathrm{SiH}_{n}\right]$ and $[\mathrm{H}]$ decomposed in the plasma when they reach the substrate surface. Therefore, the average crystalline size is adjusted by controlling the DC bias.

The transmission curve of typical sample is shown in Figure 3a. The absorption spectra and the extinction coefficient as shown in Figures $\mathbf{3 b}$ and $\mathbf{3 c}$, the interference-free regions are approximated by formula (6) and formula (7), respectively. According to the films' transmission spectra, the optical band gap can be calculated by the Wood and Tauc formula [15], as shown in Figure 3d.

$$
\begin{aligned}
& \alpha=\ln \left(\frac{1}{T d}\right) \\
& k=\frac{\alpha \lambda}{4 \pi} \\
& (\alpha h v)=B(h v-E g)^{2}
\end{aligned}
$$

Where $h v$ is the photon energy, $B$ is a ratio constant [16], $\alpha$ is the absorption coefficient.

The optical band gaps as functions of different parameters are shown in Figure 4. The optical band gap decreases from
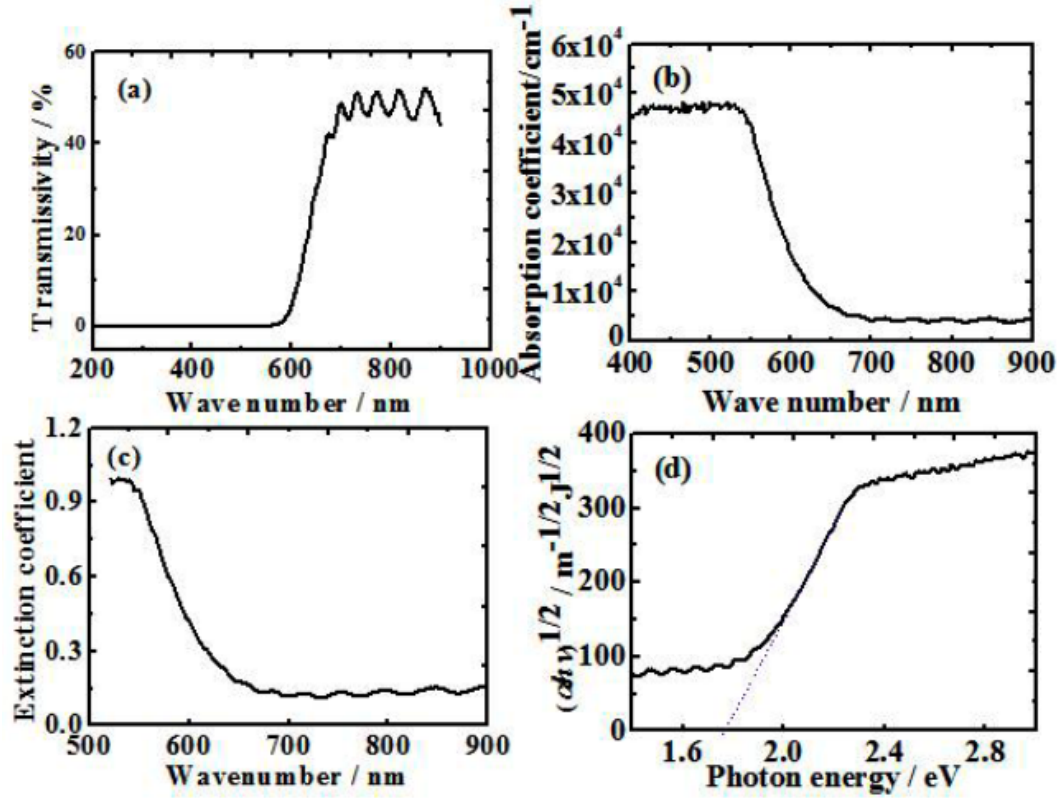

Figure 3: (a) The transmission spectra of a typical nc-Si:H thin films, (b) Absorption coefficient as a function of wavenumber, (c) Exinction coefficient as a function of wavenumber, (d) The caculated optical band gap. 

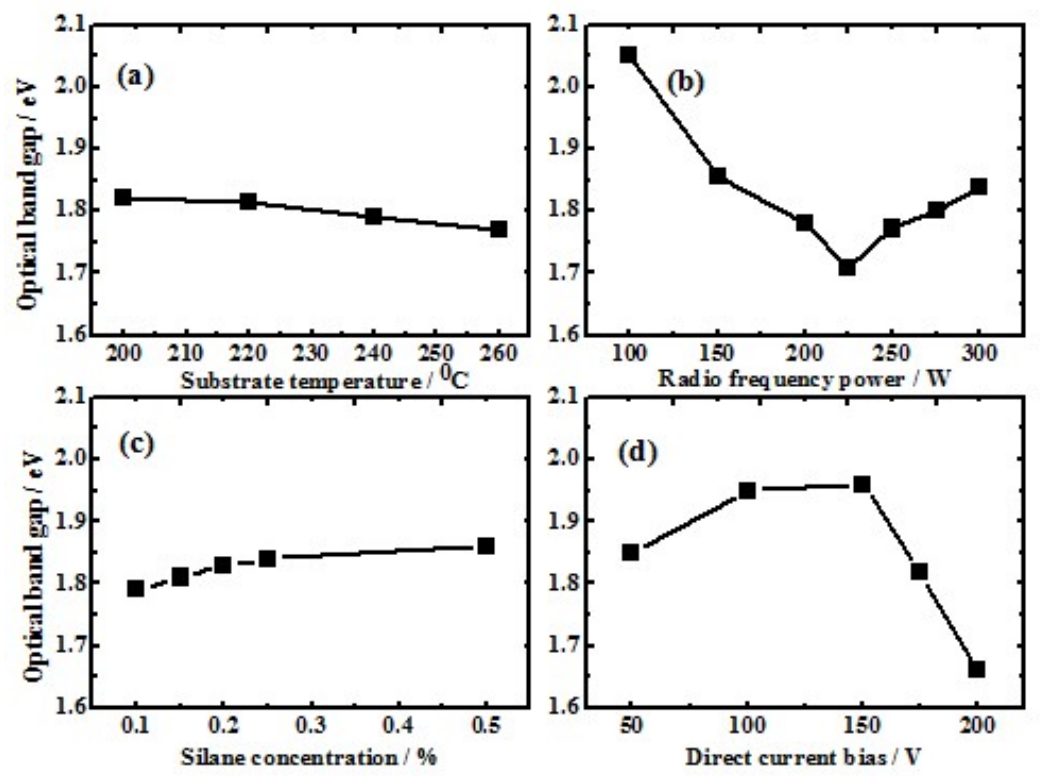

Figure 4: Multiple parameters of deposition that affect the optical band gap (a) The optical band gap as a function of substrate temperature for depositing nc-Si:H thin films, (b) The optical band gap as a function of RF power for depositing nc-Si:H thin films, (c) The optical band gap as a function of silane concentration for depositing nc-Si:H thin films, (d) The optical band gap as a function of DC bias for depositing nc-Si:H thin films.

$1.82 \mathrm{eV}$ to $1.79 \mathrm{eV}$ as the substrate temperature increases from $200{ }^{\circ} \mathrm{C}$ to $260{ }^{\circ} \mathrm{C}$, as shown in Figure 4a. With increaseing RF power from $100 \mathrm{~W}$ to $225 \mathrm{~W}$, the optical band gap decreases from $2.14 \mathrm{eV}$ to $1.75 \mathrm{eV}$. While RF power is greater than $225 \mathrm{~W}$, the optical bang gap begins to increase to $1.84 \mathrm{eV}$, as shown in Figure $\mathbf{4 b}$. This indicates that there is a threshold of the optical band gap as a function of RF power. And this result is not consistent with the result gained from samples prepared by HW-CAD [17]. With the silane concentration increases from $0.1 \%$ to $0.5 \%$, the optical band gap decreases from $1.79 \mathrm{eV}$ to $1.86 \mathrm{eV}$, as shown in Figure 4c. With increasing the DC negative bias voltage to $150 \mathrm{~V}$, the optical band gap achieved to a maximum value of 1.96 $\mathrm{eV}$. And with the DC negative bias voltage of $200 \mathrm{~V}$, the minimum value of $1.65 \mathrm{eV}$ is obtained, as shown in Figure 4d.

The optical property and the microstructure of nc-Si:H thin film are affected by the process parameters. But how can the optical property depend on the microstructure? To investigate this problem further, we showed the optical band gap as a function of the crystalline volume fraction by keeping the average crystalline size constants, as shown in Figure $\mathbf{5}$. It can be seen that the optical band gap turns out a decline trend with the increase of crystalline volume fraction. As we all know, the average crystalline size in nc-Si:H thin film is only about $2 \mathrm{~nm} \sim 8 \mathrm{~nm}$. And when the average crystalline size is closed to a certain extent, the quantum effects will happen and the absorption spectra of films will also widen. Based on the semiconductor electronic state theory, the distortion bond and the dangling bond incorporate across the band gap and band tail states density [18]. The bigger the crystalline volume fraction is, the smaller both the number of the dangling bond and the extent of the distortion bond are. The bigger the crystalline volume fraction is, the smaller both the band gap and the band tail states density are. Therefore, when increasing of the crystalline volume fraction, all of the density degree, the distortion extent and the number of dangling bonds decrease [19], resulting decreasing of the optical bang gap.

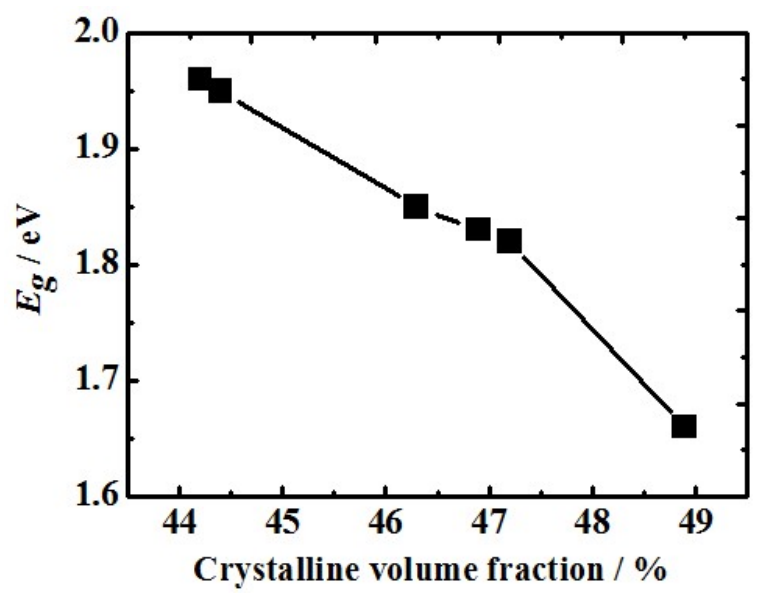

Figure 5: The optical band gap as a function of the crystalline volume fraction of nc-Si:H thin film.

\section{CONCLUSIONS}

Nc-Si:H thin film are prepared by PECVD with DC bias applied. The substrate temperature, the RF power, the silane concentration and the DC negative bias voltage are changed in turn and solely during the process of preparation. The crystalline volume fraction, the average crystalline size and the optical band gap can be optimized by appropriately 
controlling RF power, the DC negative bias voltage, the substrate temperature and the silane concentration. It was found that when keeping the average crystalline size constant and increasing the crystalline volume fraction, the optical band gap turns out a decline trend. However, due to its minimal optical band gap, nc-Si:H is an ideal material which will instead of a-Si:H films for solar cells.

\section{ACKNOWLEDGEMENT}

Project supported by the Starting Foundation of Jiangsu University Advanced Talent (14JDG049) and the National Natural Science Foundation of China (51402321).

\section{REFERENCES}

[1] Biswas R, Bhattacharya J, Lewis B, Chakravarty N, Dalal V. Enhanced nanocrystalline silicon solar cell with a photonic crystal back-reflector. Sol Energ Mat Sol C 2010; 94: 2337-42. http://dx.doi.org/10.1016/j.solmat.2010.08.007

[2] Peng YC, He YL. Research \& development on fabrications, structures and properties of nanometer silicon film materials. Chin J Rare Metals 1999; 23: 42-55.

[3] Waman VS, Kamble MM, Pramod MR, et al. Influence of the deposition parameters on the microstructure and opto-electrical properties of hydrogenated nanocrystalline silicon films by HW-CVD. J Non-Cryst Solids 2011; 357: 3616-22

http://dx.doi.org/10.1016/i.jnoncrysol.2011.07.002

[4] Swain BP, Hwang NM. Effect of negative substrate bias on HWCVD deposited nanocrystalline silicon (nc-Si) films. Solid State Sci 2009; 11: 467-71

http://dx.doi.org/10.1016/j.solidstatesciences.2008.08.004

[5] Luo PQ, Zhou ZB, Li YJ, Lin SQ, Dou XM, RQ. Effects of deposition pressure on the microstructural and optoelectrical properties of $\mathrm{B}$ doped hydrogenated nanocrystalline silicon (nc-Si:H) thin films grown by hot-wire chemical vapor deposition. Microelectron J 2008; 39: 12. http://dx.doi.org/10.1016/i.mejo.2007.10.019

[6] Liskiewicz T, Al-Borno A. DLC coatings in oil and gas production. Journal of Coating Science and Technology 2014; 1; 59-68.

[7] Ossadnik C, Vepřek S, Gregora I. Applicability of Raman scattering for the characterization of nanocrystalline silicon. Thin solid films 1999; 337: 148-51 http://dx.doi.org/10.1016/S0040-6090(98)01175-4
Guo LQ, Ding JN, Yang JC, Cheng GG, Ling ZY. Effects of high hydrogen dilution ratio on surface topography and mechanical properties of hydrogenated nanocrystalline silicon thin. Thin solid films 2011; 519: 6039-43.

http://dx.doi.org/10.1016/i.tsf.2011.04.117

[9] Wang JL, Wu EX. Characterization of doped hydrogenated nanocrystalline silicon films prepared by plasma enhanced chemical vapour deposition. Chin Phys B 2007; 16: 848. http://dx.doi.org/10.1088/1009-1963/16/3/049

[10] Zhao ZX, Cui RQ, Meng FY, Zhao BC, Yu HC, Zhou ZB. Nanocrystalline silicon thin films prepared by RF sputtering at low temperature and heterojunction solar cell. Mater Lett 2004; 58: 39636. http://dx.doi.org/10.1016/j.matlet.2004.09.004

[11] Ledinsky M, Fekete L, Stuchlik J, Mates T, Fejfar A, Kočka J. Characterization of mixed phase silicon by Raman spectroscopy. $J$ Non-Cryst Solids 2006; 352: 1209-12. http://dx.doi.org/10.1016/i.jnoncrysol.2005.10.072

[12] Wu ZM, Lei QS, Geng XH, Zhao Y, Sun J, Xi JP. Effect of substrate temperature and pressure on properties of microcrystalline silicon films. Chin Phys B 2006; 6: 1320

[13] Tong GB, Rahman A. Semiconductor Electronics, 2006. ICSE '06. IEEE International Conference, Oct. 29, 2006, Proc. Kuala Lumpur, Malaysia, p. 472-6.

[14] Lei QS, Wu ZM, Geng XH, Zhao Y, Xi JP. Influence of the deposition parameters on the transition region of hydrogenated silicon films growth. Chin Phys B 2005; 11: 2342.

[15] Wood DL, Tauc J. Weak absorption tails in amorphous semiconductors. Phys Rev B 1972; 5: 3144 http://dx.doi.org/10.1103/PhysRevB.5.3144

[16] Liao NM, LiW, Jiang YD, et al. Thickness and optical constant determination of hydrogenated amorphous silicon thin film from transmittance spectra of ellipsometer. Acta Phys Sin 2008; 57: 15427.

[17] Lien SY, Wang CC, Shen CT, et al. Effects of RF power and pressure on performance of HF-PECVD silicon thin-film solar cells. Thin solid films 2010; $24: 7233-5$. http://dx.doi.org/10.1016/i.tsf.2010.04.083

[18] Guo L, Ding J, Yang J, et al. Nanostructure, electrical and optical properties of p-type hydrogenated nanocrystalline silicon films. Vacuum 2011; 85: 649-53. http://dx.doi.org/10.1016/j.vacuum.2010.07.014

[19] Zhang ZG, Su CH. The energy dist ribution of band tall states of nc-S and it's optical gap. Acta Energiae Solaris Sinica 1996; 17: 175. 